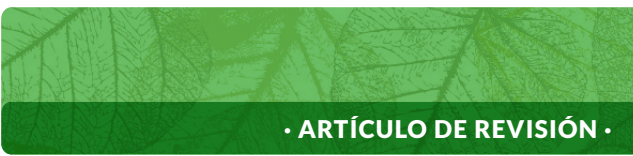

\title{
Sistemas socio-ecológicos: análisis bibliométrico del estado actual, desarrollo y escenarios futuros*
}

\section{Socio-ecological systems: bibliometric analysis of the current state, development and future scenarios}

\author{
MORALES-VELASCO, SANDRA'; RAMÍREZ-GONZÁLEZ, GUSTAVO²; \\ RAMOS-BARÓN, PABLO-ANDRÉS ${ }^{3}$; ARANGO-MEJÍA, JACOBO4; \\ VIVAS-QUILA, NELSON-JOSÉ ${ }^{5}$
}

Historial del Artículo

Recibido para evaluación: 30 de Agosto 2020

Aprobado para publicación: 17 de Septiembre 2021.

* Título Proyecto de origen: "Calculo del balance de gases de efecto de invernadero en tres agroecosistemas ganaderos del trópico seco y su aporte a la mitigación del calentamiento global”. Financiación: Tesis doctoral, Sistema General de Regalías.

1 Universidad del Cauca, Facultad de Ciencias Agrarias, Grupo de investigación NUTRIFACA. M.Sc. Ecología. Popayán, Colombia. https://orcid.org/0000-0003-2655-9366

2 Universidad del Cauca, Facultad de Ingeniería Electrónica y Telemática, Grupo de investigación Ingeniería Telemática. Ph.D. Ingeniería Electrónica y Telecomunicaciones. Popayán, Colombia. https://orcid.org/0000-0002-1338-8820

3 Pontificia Universidad Javeriana, Facultad de Estudios Ambientales y Rurales, Grupo de investigación Institucionalidad y Desarrollo Rural. Ph.D. Conflict Studies and Management. Bogotá, Colombia.

4 Centro Internacional de Agricultura Tropical, Grupo de Investigación en Forrajes Tropicales, Ph.D. Biología. Palmira, Colombia.

5 Universidad del Cauca, Facultad de Ciencias Agrarias, Grupo de investigación NUTRIFACA. Ph.D. Ciencias Pecuarias. Popayán, Colombia. https://orcid.org/0000-0003-0165-2863

Correspondencia: samorales@unicauca.edu.co

Cómo citar este artículo: MORALES VELASCO, SANDRA; RAMÍREZ-GONZÁLEZ, GUSTAVO; RAMOS-BARÓN, PABLO ANDRÉS; ARANGO-MEJÍA, JACOBO; VIVAS-QUILA, NELSON-JOSÉ. Sistemas socio-ecológicos: análisis bibliométrico del estado actual, desarrollo y escenarios futuros. Biotecnología en el sector agropecuario y agroindustrial, v. 19, n. 2, 2021, p. 251-270. Doi: https:// doi.org/10.18684/bsaa.v19.n2.2021.1747 


\section{RESUMEN}

En este documento presenta un análisis bibliométrico/mapeo científico para analizar las tendencias en la investigación de los sistemas socio-ecológicos SSE, basado en 1751 publicaciones citadas en la base de datos de Scopus entre 1971 y 2019. Para al análisis de los registros se utilizó el software SciMAT, que analiza relaciones evolución conceptual, diagramas estratégicos y clúster relacionados con la productividad de los autores, número de referencias, organizaciones, países y principales publicaciones. Los resultados muestran el incremento en estudios relacionados con los SSE, con el especial interés en el pervivir y la gobernanza de los recursos naturales. También se evidencia que los países que mayor cantidad de producción son EE. UU., Reino Unido, Australia y Alemania.

\begin{abstract}
This document presents a bibliometric analysis/scientific mapping to analyze trends in the research of socio-ecological systems - SSE, based on 1751 publications cited in the Scopus database between 1971 and 2019. The SciMAT software was used for the analysis of the records, which analyzes relations between conceptual evolution, strategic diagrams and clusters related to the productivity of the authors, number of references, organizations, countries and main publications. The results show the increase in studies related to SSE, with special interest in the survival and governance of natural resources. It is also evident that the countries with the highest amount of production are USA, UK, Australia and Germany.
\end{abstract}

\section{INTRODUCCIÓN}

El crecimiento demográfico ha generado diferentes procesos de desarroIlo, ocasionando cambios sustanciales en los componentes biogeoquímicos de la tierra, de tal manera que se habla de una nueva era geológica denominada Antropoceno o la era de los humanos (Crutzen et al., 2000), la cual se sustenta en la extinción masiva de especies, variaciones en el paisaje natural y la agricultura moderna. Toma como punto de partida la revolución industrial (1800), pasando por la aparición de los radioisótopos (producto de las bombas atómicas de los años 40 y 50), hasta el cambio climático global (Ruddiman and Kutzbach, 1991). Lo anterior es explicado (Hardin, 1968) en el documento "La tragedia de los Comunes", que describe una situación en la cual diferentes personas, motivados solo por el interés personal, sobreexplotan un recurso compartido limitado (el común: agua, suelo etc.), aunque a ninguno de ellos les convenga que tal destrucción suceda.

La demarcación de una nueva época (Antropoceno) trae consecuencias en el ámbito práctico y legal, ya que eventos mundiales como la pandemia (Covid 19), conlleva a regulaciones en las leyes internacionales sobre los cambios de las instituciones humanas y en particular en el manejo de las especies silvestres, dada la presunción del origen zoonótico del virus (Cruz et al., 2020); donde científicos señalan que la invasión del mundo

\section{PALABRAS CLAVE:}

Análisis bibliométrico; Mapeo; Bases de datos; Sistemas socio-ecológicos; Gobernanza; Perspectivas.

\section{KEYWORDS:}

Bibliometric analysis; Mapping; Databases; Socio-ecological systems; Governance; Perspectives. 
natural origina alteraciones en las relaciones entre el hombre y el ecosistema, precisando la adaptación a un nuevo ordenamiento para incorporar esos cambios crecientes.

En el documento "El Gobierno de los bienes comunes" (Ostrom, 1990), propone la gestión colectiva de los recursos naturales, base del desarrollo de sistemas socio-ecológicos sostenibles, ya que permiten comprender las interacciones entre los diferentes componentes del objeto del estudio (industria, agricultura, ganadería, ruralidad, urbano, hombre, ambiente, etc.) y las posibles prácticas de retroalimentación que contribuyan con la resiliencia basada en prácticas (culturales, políticas, ambientales) propias de unas condiciones agroecológicas de un país o región (Ostrom, 2009).

Los sistemas socio-ecológicos - SES, se basan en la ecología y la teoría de la complejidad, bajo problemáticas sociales como el bienestar humano, que pocas veces es incluido en los sistemas adaptativos complejos (Cumming, 2011), incorporando aspectos concernientes a la resiliencia, sostenibilidad y vulnerabilidad (Levin, 2001; Holling and Gunderson, 2002).

Los análisis bibliométricos tradicionales se basan en indicadores de rendimiento de las publicaciones, relacionando la producción científica con las contribuciones a la cuantificación del conocimiento (Zachos, 1991). Se dividen en dos grupos: los que evalúan los recursos (instrumentos, financiación, infraestructura, etc.) y los de resultados científicos (calidad, actividad científica, conexión, citaciones, impacto de la fuente, asociación temática) (Sancho, 1990).

De esta manera, se realizó un análisis bibliométrico con el objetivo de identificar las tendencias en la investigación basados en sistemas socio ecológicos.

\section{DESARROLLO DEL TEMA}

La presente revisión es un estudio bibliográfico a partir de registros extraídos de la base de datos Scopus; las publicaciones seleccionadas para el análisis fueron artículos de revistas citadas, libros, conferencias, documentos de trabajo, tesis e informes que enumeran la frase "Sistemas Socio Ecológicos"

\section{Herramienta de análisis}

Se utilizó el software SciMAT (Sciencie Mapping Analysis Software Tool), herramienta de código abierto para el mapeo científico, representa gráficamente las relaciones de evolución conceptual, diagramas estratégicos y clústers entre los documentos publicados.

\section{Configuración del análisis}

El tiempo de observación fue desde 1971 hasta 2019, es decir, desde el año en que el término de "Sistemas Socio Ecológicos" fue usado por primera vez (Joun, 1971) hasta el más reciente. Teniendo en cuenta los eventos relacionados con el tema, se consolidaron tres periodos:

1971-2000: se resaltan problemáticas ambientales publicadas en diferentes documentos (Carson, 1963; Commoner, 1977; Commoner, 1978; Schumacher, 1978) y acuerdos establecidos por la ONU (Educación Ambiental 1968; Informe de Roma 1972; Programa de las Naciones Unidas para el Medio Ambiente PNUMA 1973; Cumbre de Río 1992: Conferencia de la Partes para el Cambio Climático -1995; Protocolo de Kioto 1997; Declaración del Milenio 2000) (Organización de las Naciones Unidas ONU, 1998; ONU, 2000). 
2001-2010: promueve el desarrollo sostenible y la calidad de vida de las personas que viven en pobreza - Buen Vivir; y revertir la degradación medio ambiental y el cambio climático (Nationally Appropriate Mitigation Actions (NAMAs, Johannesburgo, 2002) (Tewari, 2013).

2011-2019: aprobación de la agenda 2030 y acuerdo de Paris (2015), los objetivos de desarrollo sostenible (Fernández, 2016). En la configuración del Scimat se utilizó la configuración presentada en el cuadro 1.

Cuadro 1. Parámetros de configuración de SciMAT.

\begin{tabular}{|l|l|}
\hline \multicolumn{1}{|c|}{ Parámetro } & \multicolumn{1}{c|}{ Descripción } \\
\hline Unidad de análisis & Palabras (autor Rol = verdadero, fuente Rol = verdadero, rol añadido = verdadero). \\
\hline Tipo de red & Co-ocurrencia \\
\hline Medida de normalización & Índice de equivalencia - Índice h \\
\hline Algoritmo del clúster & Centros simples (Tamaño máximo del clúster: 7 - Tamaño mínimo del clúster: 3) \\
\hline Medida de la evolución & Índice de inclusión \\
\hline Medida superpuesta & Índice de inclusión \\
\hline
\end{tabular}

Datos obtenidos. Se registraron 1751 artículos en la base de datos de Scopus, donde se observa un incremento a partir del último periodo de análisis (2011-2019), que concuerda con los procesos sobre resiliencia y desarroIlo sostenible reglamentado por la ONU.

La revista que registro mayores publicaciones referentes a los SSE fue Sustainability, donde el objetivo principal es la economía social y el análisis de las personas, planeta, prosperidad, paz y cooperación (cuadro 2).

Cuadro 2. Principales revistas que registran publicaciones en Sistemas Socio Ecológicos.

\begin{tabular}{|c|c|c|c|c|c|}
\hline Revista & $\begin{array}{c}\text { Cobertura de } \\
\text { la revista }\end{array}$ & $\begin{array}{l}\text { Número de } \\
\text { documentos }\end{array}$ & Área & \begin{tabular}{|c|}
$\begin{array}{c}\text { Factor de impacto } \\
\text { (2018 JCR) }\end{array}$ \\
\end{tabular} & $\begin{array}{c}\text { Factor de } \\
\text { impactos- } 5 \text { años }\end{array}$ \\
\hline $\begin{array}{l}\text { Sustainability } \\
\text { (Switzerland) }\end{array}$ & 2009 & 58 & $\begin{array}{l}\text { Energy } \\
\text { Environmental science } \\
\text { Management } \\
\text { Policy and law } \\
\text { Social sciences } \\
\end{array}$ & 2,592 & 2,801 \\
\hline $\begin{array}{l}\text { Science of the Total } \\
\text { Environment }\end{array}$ & $1972-2019$ & 38 & $\begin{array}{l}\text { Environmental science } \\
\text { Environmental chemistry }\end{array}$ & 5,589 & 5,727 \\
\hline Ecological Economics & 1989 & 31 & $\begin{array}{l}\text { Economics } \\
\text { Environmental science } \\
\text { (miscellaneous) }\end{array}$ & 4,281 & 5,207 \\
\hline $\begin{array}{l}\text { Environmental Science } \\
\text { and Policy }\end{array}$ & 1998 & 29 & $\begin{array}{l}\text { Environmental science } \\
\text { Social sciences } \\
\text { Geography }\end{array}$ & 4,816 & 5,127 \\
\hline Ecological Indicators & 2001 & 28 & $\begin{array}{l}\text { Agricultural and biological } \\
\text { sciences } \\
\text { Decision sciences (miscella- } \\
\text { neous) }\end{array}$ & 4,49 & 4,863 \\
\hline Land Use Policy & 1984 & 25 & $\begin{array}{l}\text { Agricultural and biological } \\
\text { sciences } \\
\text { Forestry } \\
\text { Environmental science } \\
\text { Social sciences }\end{array}$ & 3,573 & 4,236 \\
\hline
\end{tabular}




\begin{tabular}{|l|l|l|l|l|l|}
\hline \multicolumn{1}{|c|}{ Revista } & $\begin{array}{l}\text { Cobertura de } \\
\text { la revista }\end{array}$ & \multicolumn{1}{|c|}{$\begin{array}{c}\text { Número de } \\
\text { documentos }\end{array}$} & \multicolumn{1}{c|}{ Área } & $\begin{array}{l}\text { Factor de impacto } \\
\text { (2018 JCR) }\end{array}$ & $\begin{array}{c}\text { Factor de } \\
\text { impactos- 5 años }\end{array}$ \\
\hline $\begin{array}{l}\text { Global Environmental } \\
\text { Change }\end{array}$ & 1990 & 24 & $\begin{array}{l}\text { Environmental science } \\
\text { Ecology } \\
\text { Social sciences } \\
\text { Geography }\end{array}$ & 10,427 & 11,223 \\
\hline $\begin{array}{l}\text { Journal of Environmen- } \\
\text { tal Management }\end{array}$ & 1976 & 23 & $\begin{array}{l}\text { Environmental science } \\
\text { Waste Management and } \\
\text { disposal } \\
\text { Medicine (miscellaneous) }\end{array}$ & 4,865 & 4,92 \\
\hline PLoS ONE & 2006 & 22 & $\begin{array}{l}\text { Agricultural and biological } \\
\text { sciences (miscellaneous) } \\
\text { Biochemistry, genetics and } \\
\text { molecular biology (miscella- } \\
\text { neous) } \\
\text { Medicine (miscellaneous) }\end{array}$ & 2,7776 & 3,534 \\
\hline $\begin{array}{l}\text { Environmental Mode- } \\
\text { Iling and Software }\end{array}$ & 2014 & 21 & $\begin{array}{l}\text { Computer science } \\
\text { Environmental science } \\
\text { Ecological modeling }\end{array}$ & 4,552 & 5,093 \\
\hline Ecological Modelling & 1975 & $\begin{array}{l}\text { Environmental science } \\
\text { Ecological modeling }\end{array}$ & 2,634 & 2,852 \\
\hline
\end{tabular}

Los autores con mayor número de documentos publicados se registran en el cuadro 3. Las principales temáticas presentan resultados de investigaciones acerca del uso del suelo, la resiliencia y el impacto socioeconómico sobre las comunidades.

Cuadro 3. Autores con mayores publicaciones en Sistemas Socio-ecológicos.

\begin{tabular}{|l|c|c|}
\hline \multicolumn{1}{|c|}{ Autor } & No. documentos & Periodo \\
\hline Salvati, L. & 13 & $2014-2019$ \\
\hline Verburg, P.H. & 12 & $2006-2019$ \\
\hline Krausmann, F. & 9 & $2006-2019$ \\
\hline van Putten, I. & 8 & $2014-2019$ \\
\hline Zurlini, G. & 8 & $2006-2019$ \\
\hline Petrosillo, I. & 7 & $2006-2019$ \\
\hline Qureshi, S. & 7 & $2014-2018$ \\
\hline Haberl, H. & 7 & $2006-2016$ \\
\hline Schmitz, M.F. & 6 & $2008-2019$ \\
\hline Bardsley, D.K. & 6 & $2012-2016$ \\
\hline
\end{tabular}

Cinco de los autores publican a partir del 2006, acorde con la entrada en vigor del protocolo de Kyoto (ONU, 1998), el cual propende con lineamientos para el desarrollo sostenible, entre los que se resaltan:

- Gestión del uso de la tierra y la silvicultura.

- Desarrollo y aplicación de procesos ecológicamente racionales.

- Reducción de efectos adversos del cambio climático.

Artículos más citados. La publicación más citada registra 1069 (Gallopín, 2006); donde la importancia radica en la utilización de un modelo sistémico para identificar y analizar las relaciones conceptuales entre la vulnerabilidad, la resistencia y la adaptación al cambio (Cuadro 4). 
Cuadro 4. Autores con mayores citaciones en Sistemas Socio-ecológicos.

\begin{tabular}{|l|l|c|c|}
\hline \multicolumn{1}{|c|}{ Articulo } & \multicolumn{1}{|c|}{ Autor } & Año & \multicolumn{1}{|c|}{ Cit. } \\
\hline $\begin{array}{l}\text { Linkages between vulnerability, resilience, and adaptive } \\
\text { capacity }\end{array}$ & Gallopín, G.C. & 2006 & 1069 \\
\hline $\begin{array}{l}\text { Land use transitions: Socio-ecological feedback versus } \\
\text { socio-economic change }\end{array}$ & Lambin, E.F.; Meyfroidt, P. & 2010 & 438 \\
\hline $\begin{array}{l}\text { The globalization of socio-ecological systems: An agenda } \\
\text { for scientific research }\end{array}$ & $\begin{array}{l}\text { Young, O.R., Berkhout, F., Gallopin, G.C., } \\
\text { Janssen, M.A., Ostrom, E., van der Leeuw, S. }\end{array}$ & 2006 & 437 \\
\hline $\begin{array}{l}\text { Resilience thinking meets social theory: Situating social } \\
\text { change in socio-ecological systems (SES) research }\end{array}$ & Cote, M., Nightingale, A.J. & 2012 & 427 \\
\hline $\begin{array}{l}\text { Knowing food and growing food: Beyond the produc- } \\
\text { tion-consumption debate in the sociology of agriculture }\end{array}$ & Goodman, D., Du Puis, E.M. & 2002 \\
\hline $\begin{array}{l}\text { Resilience of past landscapes: Resilience theory, society, } \\
\text { and the Longue DurÃ@e }\end{array}$ & Redman, C.L., Kinzig, A.P. & 253 \\
\hline $\begin{array}{l}\text { Zero-Sum world: Challenges in conceptualizing environ- } \\
\text { mental load displacement and ecologically unequal exchan- } \\
\text { ge in the world-system }\end{array}$ & Hornborg, A. & 2003 & 199 \\
\hline Backcasting â€” a framework for strategic planning & Holmberg, J., Robert, K.-H. & 2009 & 193 \\
\hline $\begin{array}{l}\text { "Stationarity is dead"- Long live transformation: Five princi- } \\
\text { ples for climate change adaptation law }\end{array}$ & Craig, R.K. & 2000 & 186 \\
\hline $\begin{array}{l}\text { Overcoming systemic roadblocks to sustainability: The } \\
\text { evolutionary redesign of worldviews, institutions, and } \\
\text { technologies }\end{array}$ & $\begin{array}{l}\text { Beddoe, R., Costanza, R., Farley, J., Garza, } \\
\text { E., Kent, J., Kubiszewski, l., Martinez, L., } \\
\text { McCowen, T., Murphy, K., Myers, N., Ogden, } \\
\text { Z., Stapleton, K., Woodward, J. }\end{array}$ & 2009 & 182 \\
\hline
\end{tabular}

Investigaciones introducen el concepto de transición, asociado con factores de cambio entre la sociedad y en el sistema biofísico, que surgen del agotamiento de los recursos (Lambin and Meyfroidt, 2010). Otros autores realizan análisis de los efectos de la globalización (megatendencias, megaciudades), la vulnerabilidad y adaptabilidad, concluyendo que los SES pueden conducir a iniciativas para evitar o mitigar los impactos (Young et al., 2006).

Palabras clave. Los resultados del Scimat muestran 2190 palabras clave; las más registradas se consignan en el cuadro 5.

Cuadro 5. Principales palabras claves usadas en publicaciones de Sistemas Socio-ecológicos.

\begin{tabular}{|l|c|}
\hline \multicolumn{1}{|c|}{ Palabra clave } & Número de documentos \\
\hline Climate-Change & 250 \\
\hline Ecology & 244 \\
\hline Sustainability & 237 \\
\hline Sustainable-Development & 226 \\
\hline Ecosystems & 199 \\
\hline Water-Management & 194 \\
\hline Resilience & 191 \\
\hline Socioeconomic-Conditions & 183 \\
\hline Conservation & 177 \\
\hline Decision-Making & 171 \\
\hline
\end{tabular}

Las publicaciones muestran articulación entre estrategias para la mitigación del cambio climático y la gobernanza de los recursos naturales, para la estabilidad de las condiciones socioeconómicas del ser humano. 


\section{Variación en el tiempo de las publicaciones relacionadas con Sistemas Socio-ecológicos}

En el primer periodo, que abarca un espacio de 29 años, se registraron 54 artículos, evolucionando para los dos siguientes a 208 y 1489 publicaciones. Es de resaltar que el último tiempo concentra el 85,03\% de los papers escritos, lo que evidencia un interés general por las investigaciones relacionadas con el tema. Las palabras clave evolucionaron en relación con el número de artículos publicados, donde se señalaron 89, 415 y 409 para cada periodo (Figura 1).

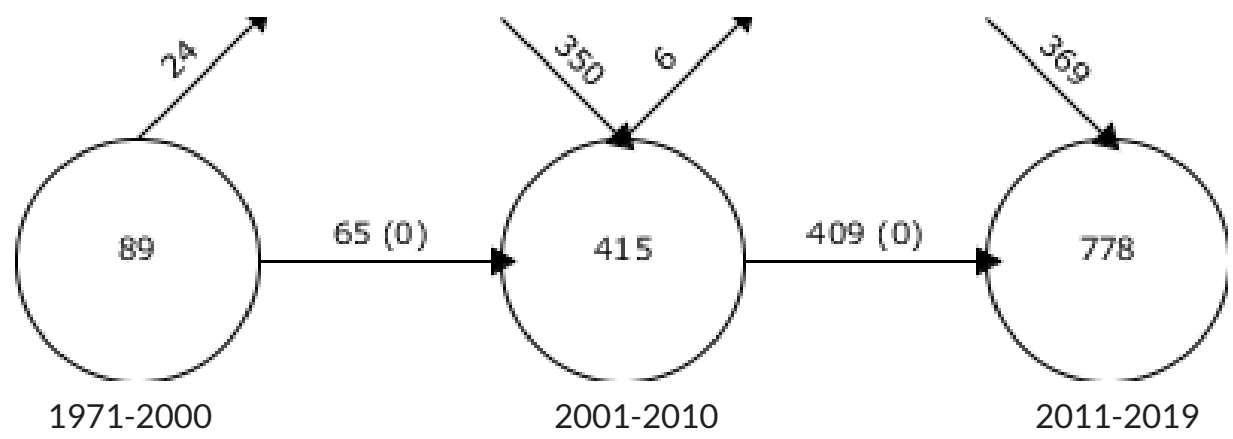

Figura 1. Número de palabras clave traslapadas en el mapa de publicaciones sobre sistemas Socioecológicos.

Entre 1971-2000 se presentan 89 palabras, para el siguiente intervalo de tiempo (2001-2010) no reaparecen 24, migran 65 y se reconocen 350 nuevas. El último periodo (2011-2019) está caracterizado por excluir 6 términos, trasladando 409 e incluyendo 369 diferentes.

El número de palabras claves adicionadas en cada periodo muestran un incremento del 84,3 y $47,4 \%$ respectivamente, evidenciando un crecimiento de la temática de investigación; concordando con las tendencias propuestas en las publicaciones del primer y segundo periodo, en donde se establecen herramientas de diagnóstico en relación con las dimensiones naturales y sociales del cambio mundial (Gallopín, 2006; Ostrom, 2009; Ostrom, 2011). En la figura 2 se presenta la evolución de los artículos publicados en relación a los sistemas socio-ecológicos, en donde las líneas representan vínculos conceptuales, mientras las de puntos azules comparten palabras clave.

Los temas que se grafican con mayor tamaño son los que presentan un índice $\mathrm{h}$ superior; los conceptos que registran una complementariedad o madurez en las temáticas para los tres periodos.

Se resaltan las siguientes agrupaciones:

INDICATORS - ENVIROMENTAL CHANGE - CLIMATE CHANGE, base conceptual para las evaluaciones ambientales y mediciones de impactos de las actividades antrópicas.

LAND MANAGEMENT - POLICY MAKING - CLIMATE CHANGE, evidencia el manejo en el uso de la tierra y los procesos para la generación de políticas.

HUNTER GATHERER - SOCIO ECOLOGICAL, asiento de la sociedad y del desarrollo de los sistemas socio ecológicos en el manejo.

SUSTENTABILITY - SUSTENTABILITY DEVELOPMENT, herramienta del manejo de los recursos naturales para la sostenibilidad de las comunidades. 
POVERT- SOCIOENOMIC CONDICIONS - WATER RESOURCE, el agua como un indicador de pobreza y condiciones socioeconómicas.

Otro aspecto a resaltar es la transformación de las palabras clave, evidenciando como un terminó impacta la investigación hacia otros aspectos. Por ejemplo, LIVESTOCK, se expande hacia ENVIROMENTAL PROTECTION y FARMING SYSTEM.

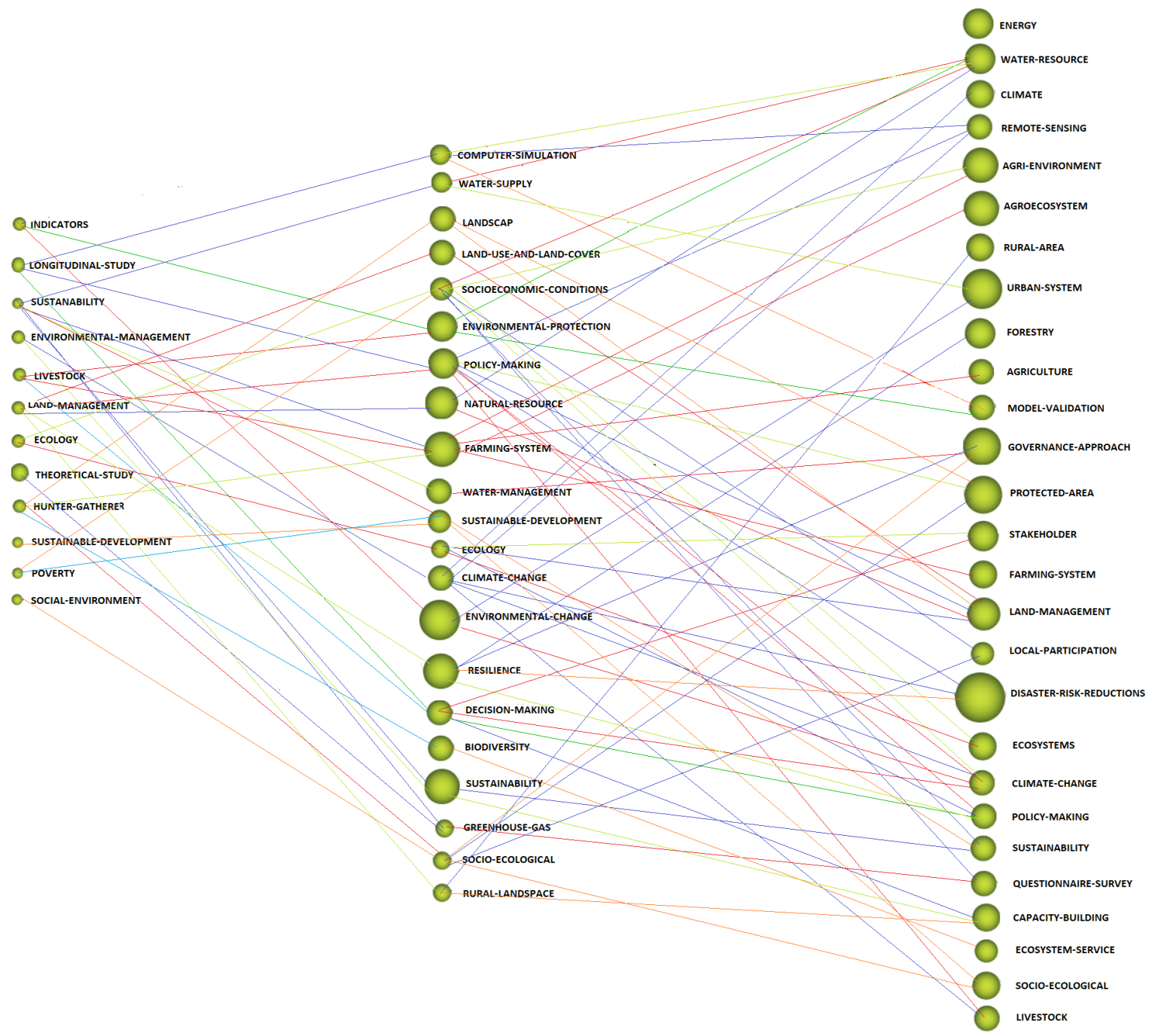

Figura 2. Evolución del mapa conceptual por periodos.

Los tópicos entre periodos se caracterizan por desarrollarse en diferentes ejes, así:

Metodológicos. Para el primer periodo se destacan palabras como INDICATORS, LOGITUDINAL-STUDY, THEORETICAL-STUDY; las publicaciones documentan lineamientos para elaborar evaluaciones ecológicas y económicas integradas, como herramienta facilitadora de adopción de decisiones y el desarrollo de una sociedad (Crook et al,. 1976; Nilsson and Bergstrom, 1995; Azar et al., 1996). 
En el segundo periodo Policy Making, Decision-Making, Computer-Simulation; identifican variables de evaluación social que determinan la formulación de políticas y la gobernanza de los recursos naturales (He et al., 2010; Sessa and Ricci, 2010; Fulton et al., 2011; Travers et al., 2011), para generar modelos de las actividades humanas sobre el manejo de los ecosistemas (Matthews et al., 2005; Syme et al., 2009; Cherkashin and Leshchenko, 2010).

Para el último se resaltan conceptos como Remote-Sensing, en estudios sobre cambio climático, fenómenos atmosféricos y cambios en el uso de la tierra (Verbesselt et al., 2012; Knox et al., 2013; Baudouin et al., 2014; Davies et al., 2016); Model Validation, usado para pronosticar comportamientos de plagas y de cultivos, como también procesos de desarrollo en una comunidad en las actividades agroalimentarias (Crespo et al., 2011; Martínez et al., 2013; Verhoog et al., 2016; Coston et al., 2017). Questionnaire-Survey herramienta de recolección de información primaria en publicaciones con enfoque social, que buscan la percepción comunitaria hacia el uso, manejo y conservación de los ecosistemas (Babai et al., 2015; Ciftcioglu, 2017; Shooshtarian and Rajagopalan, 2017; Acosta et al., 2018; Mckenzie et al., 2018; Nunes et al., 2019; Dang et al., 2019).

Unido a los anteriores, se resalta Capacity-Building, instrumento de ordenamiento de los SSE que exalta la capacidad gestora de la comunidad para la creación de estrategias de prevención de desastres y la conformación de acuerdos interinstitucionales (Cummins and Mckenna, 2010; Ruiz and Gual, 2012; Negi and Maikhuri, 2013; Alcayna et al. 2016; Hong et al., 2017; Vahanvati, 2018; Mahmood and Shaghaghi, 2019).

Ecológicos y ambientales. Entre 1971 y 2000, el término de Environmental-Management, explora paradigmas relacionadas con políticas aplicadas a modelos socio ecológicos, para evaluar el ordenamiento ambiental y disminuir los conflictos entre comunidades locales, frontera agrícola y vida silvestre (Thomson, 1993; Naughton, 1999; James et al., 2000). Land-Management, conceptualiza la visión de grupos sociales en relación a la degradación del paisaje; dado a que el conocimiento local es importante para especificar las condiciones de vida y la vulnerabilidad funcional regional-geográfico (Ellen, 1978; Nilsson and Bergstrom, 1995; Plut, 1995; James, 2000). La palabra Ecology se desarrolla en investigaciones sobre la economía de subsistencia de productores a pequeña escala, que han usado modelos ecológicos basados en bioenergética (Ellen, 1978; Carlson et al., 2000).

Para las publicaciones registradas entre los años 2001-2010 relaciona Water-Supply, resalta la importancia del recurso en diferentes escalas (energética, social y productiva) (Beddoe et al., 2009; Mcnally et al., 2009; Torres et al., 2009), donde la acción colectiva del agua se consolida como base de la sustentabilidad de las cadenas productivas con la frase Water- Management (Araral, 2009).

Otros términos que se prevalecen son Natural-Resource y Land-Use-And-Land-Cover concentrados en pesquisas de paisajes rurales y los efectos en las escalas ecológicas (Ramakrishnan, 2003; Willebrand et al., 2006; Garmendia and Stagl, 2010). También se denotan palabras como Biodiversity, Climate-Change y Greenhouse-Gas, concernientes a disertaciones sobre el uso de la biodiversidad en la producción agropecuaria para mitigar los efectos adversos del calentamiento global (Donadieu, 2002; Bardsley, 2003).

Para los años 2001-2019 son reiterativos conceptos como: Water-Resource, Climate-Change Land-Management y se reconocen nuevos Foresty, Protected-Area, Ecosystems, Ecosystem-Service y Disaster-Risk-Reductions, que identifican servicios suministrados por los ecosistemas naturales y que sustentan el desarrollo de las actividades vitales del ser humano (Dwyer and Minnegal, 1991; Owethu and Buschke, 2019).

Socioeconómicos. Se manifiestan palabras como Sustainability, Hunter-Gatherer, Poverty, Social-Environment (1971 a 2000), en estudios de "regímenes socio-metabólicos" bajo transiciones comparativas: cazadores-recolectores, sociedades agrarias-sociedad industrial; trazan desafíos hacia principios de sociedad y economía, desarrollando flujos bajo el potencial uso de la tierra; promocionando de esta manera la eficiencia ecológica (Dwyer and Minnegal, 1991; Kaufman, 1992). 
Para el siguiente periodo (2001-2010) se registran Socioeconomic - Conditions, Sustainable - Development, Resilience, Rural - Landspace, estrategias de gobernanza que permiten disminuir la pobreza e incrementar el desarrollo sostenible de las comunidades (Hodge, 2007; Jiao and Liang, 2012).

Entre el 2011 y el 2019 se destacaron los conceptos Urban-System, Governance-Approach, Stakeholder, Local-Participation, en investigaciones de producción de alimentos y sus consecuencias hacia el cambio climático, para lo cual proponen energías limpias y el fortalecimiento de la cadena de valor.

Productivo. En el primer periodo Livestock, se halla en publicaciones sobre la cría de ganado bovino que analiza la sostenibilidad de los sistemas de producción (Oro, 2000). Para el 2001-2010, se registra el termino Farming-System, describe estudios que muestran funciones, estructuras y el grado de autoorganización (aprendizaje y adaptación) para la resiliencia y sostenibilidad de la producción (Milestad and Darnhofer, 2003; Girardin et al., 2004; Vityakon et al., 2004).

Para el tercer periodo (2011-2019), se evidencia una especificidad en las publicaciones donde se categorizan como Agri-Environment, Agroecosystem, Agriculture, Farming-System y Livestock, que abordan estudios que intentan comprender cada componente del ecosistema como la parte de un todo, integrándolo de forma sinérgica para consolidar una productividad sustentable (Bardsley and Bardsley, 2014; Babai et al., 2015; Frueh et al., 2018; Waylen et al., 2019).

Temas de investigación. Los diagramas estratégicos proporcionan el número de registros a cada tema desarrollado. A continuación, se mostrarán de acuerdo a cada periodo.

1971-2000. En la figura 3 se observan los temas emergentes que han determinado el desarrollo de las investigaciones en sistemas socio-ecológicos.
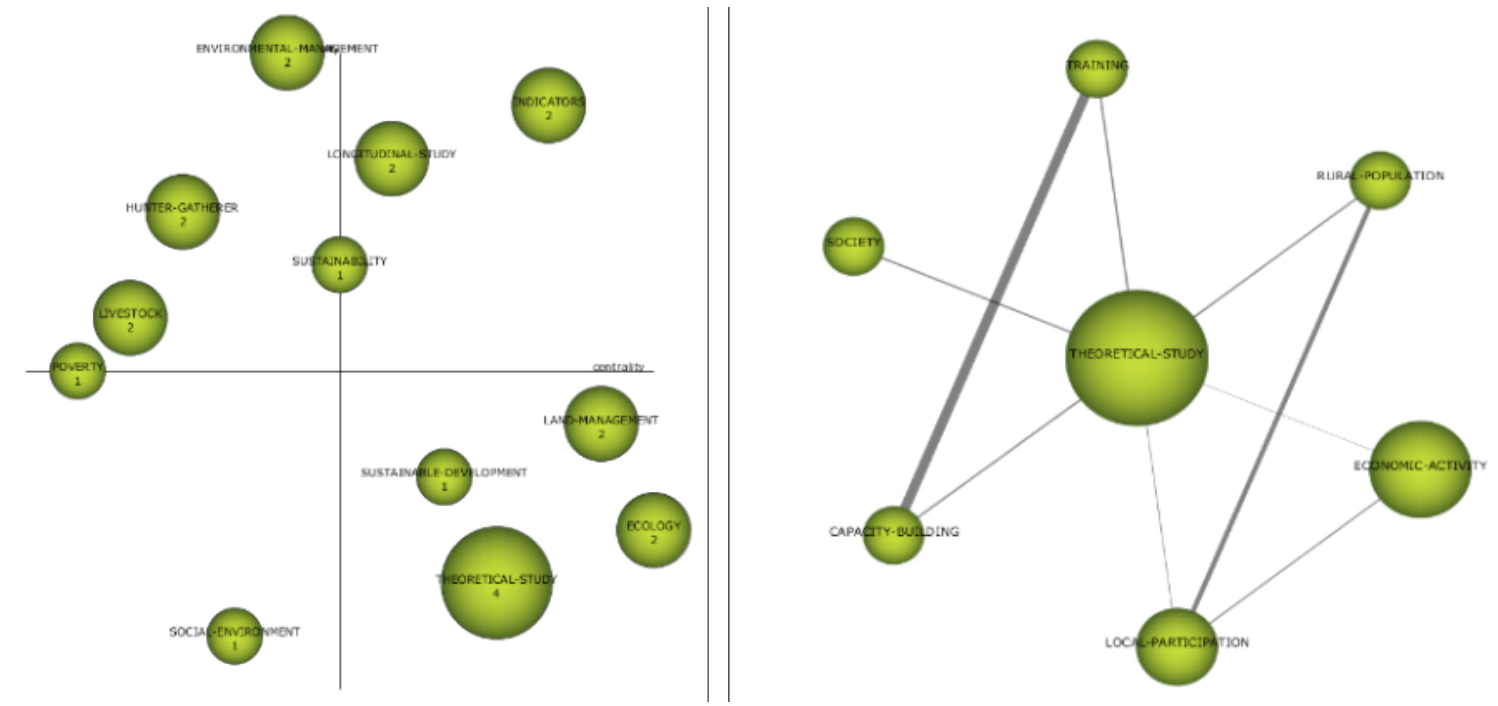

Figura 3. A. Diagrama estratégico; B. Clúster de información para el primer periodo de análisis

Se puede observar como el término emergente Social-Enviroment describe las jerarquías estructurales entre niveles a diferentes escalas temporales y espaciales (Gallopin, 1991; Sword, 1999).

El termino con mayores publicaciones fue Theoretical-Study, revela la participación local en relación con los cambios en los procesos paisajísticos-ecológicos (White, 1988; Thomson, 1993; Grove and Edwards, 1993; Plut, 1995). 
2001-2010. Para el segundo periodo se registran seis conceptos en el cuadrante emergente: Ecology, Socio-Ecological. Sustainable-Development, Water-Managment, Sustanability y Resilience (Figura 4).

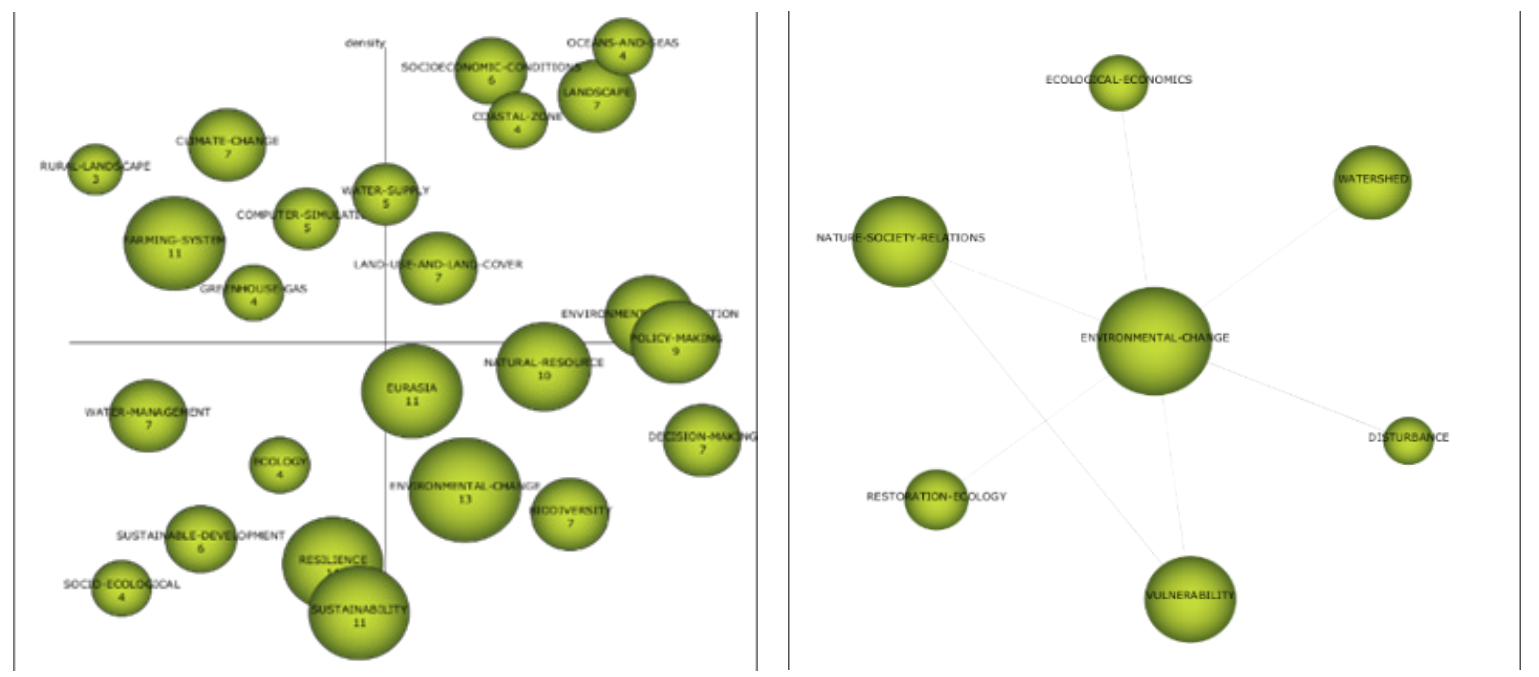

Figura 4. A. Diagrama estratégico; B. Clúster de información para el segundo periodo de análisis.

El termino Socio-Ecological, consolidado bajo la premisa de entender las relaciones entre los recursos naturales y la gobernanza mediante la teoría general de sistemas, que intenta explicar que las partes funcionan como un todo (Western, 2004; O’Neal, 2005; Barton and Haslett, 2007; Macleod, 2010).

Resilience propone métodos para entender la adaptación, funciones del ecosistema y cambios institucionales que se pueden originar en los SSE (Gallopin, 2010). Enviromental-Change registra 13 publicaciones; que refieren sobre indicadores ecológicos para la restauración de los ecosistemas.

2011- 2019. En este periodo se concentran la mayor cantidad de artículos y el comportamiento de las temáticas se dieron de la siguiente manera (Figura 5).

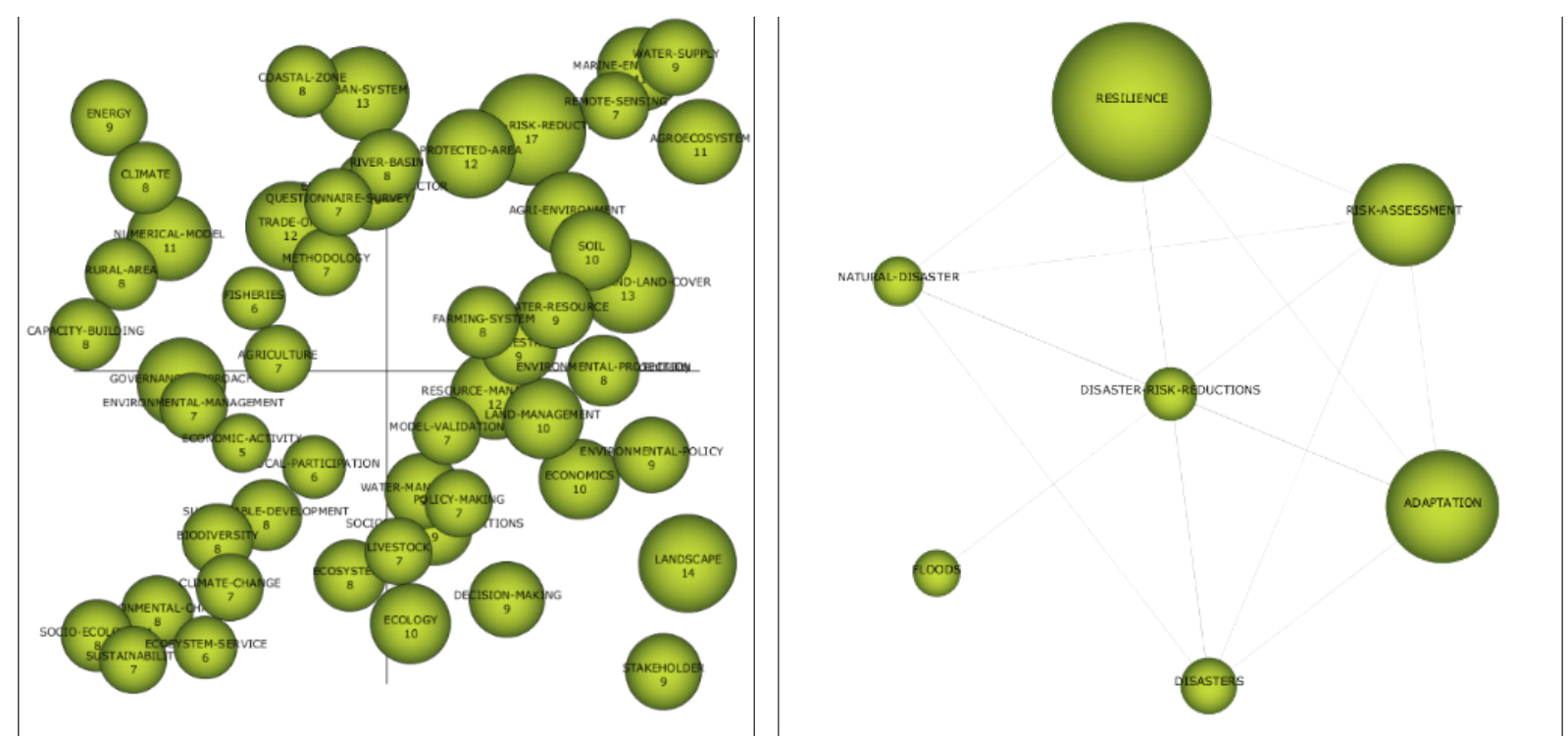

Figura 5. A. Diagrama estratégico; B. Clúster de información para el tercer periodo de análisis. 
Governance-Approach, tópico emergente en el cual se basan los SES (12 documentos), debido a la gobernanza en torno a los procesos de desarrollo sostenible de un territorio o región. Local-Participacion, propone lineamientos para la formulación de las políticas públicas. Climate-Change y Ecosystem Service, tópicos promovidos por la ONU con el propósito de propender un manejo ambiental de los ecosistemas naturales. Disaster-Risk-Reductions se localiza en el cuadrante motor, relacionada con la adaptación y resiliencia, explicado a la estabilidad buscada por los SSE.

Es así, como los sistemas socio ecológicos se fortalecen como una tendencia de desarrollo sustentable, en la cual hay una articulación entre el sector primario y el consumidor final para la estabilidad productiva; sin embargo, es necesaria la consolidación de la agroindustria rural como elemento transformador de la cadena de valor, con el fin de asegurar la rentabilidad hacia los campesinos y la eficiencia en cada uno de los eslabones, lo que incrementará la resiliencia en el suministro de alimentos, en particular durante la emergencia sanitaria que atraviesa toda la humanidad (Burkart et al., 2020; Comisión Económica para América Latina y el Caribe CEPAL, 2020).

Otro efecto evidente en la consolidación de los SSE, es el empoderamiento femenino y la igualdad de género, ya que este tipo de sistemas van de la mano con la articulación de políticas públicas y en particular con los objetivos de desarrollo sostenible, en el cual se propende en "Alcanzar la igualdad entre los géneros y empoderar a todas las mujeres y niñas". De esta manera la perspectiva socio-ecológica feminista estudia la gestión, conservación y utilización de la agrobiodiversidad, caracterizada bajo cuatro enfoques: análisis de las instituciones, conocimientos locales, economía doméstica y cadenas de productos básicos; donde las mujeres no solo cumplen su labor inherente de madre y esposa; sino también son gestoras de la producción, proveen alimento a sus hijos (madres solteras) y de la conservación de la biodiversidad de su territorio (Padmanabhan, 2011).

Otra relevancia expresada en las publicaciones, son los procesos de gobernanza y las funciones de la organización cooperativa, los cuales permiten salvaguardar los valores culturales locales, administración del paisaje, conservación de la biodiversidad y desarrollo regional (Lutz, 2013; Bardsley and Bardsley, 2014; World Health Organization WHO, 2020). Es así como metodologías socio-ecológicas han contribuido en el conocimiento y conservación de la agrodiversidad por los agricultores, que identificaron factores que influyen en la producción y el rendimiento, disminuyendo la dependencia de granos externos a conservados en la granja o redes sociales, condición que fortalece la promoción de empresas de los agricultores y las prácticas de adaptación de los cultivos a escala regional (Kansime and Mastenbroek, 2016).

Estudios afirman que el $75 \%$ de todas las enfermedades infecciosas se transmiten de animales a personas, las cuales se dan por consumo de animales silvestres, agricultura mono-especializada, malas prácticas de bioética y fragmentación de los hábitats que obligan a las especies a migrar hacia núcleos urbanos (Bardsley, 2003; ONU, 2020), alteraciones potencialmente reversibles, mediante prácticas de conservación de suelo y estrategias agropecuarias sostenibles (Agroforestería, Agricultura orgánica, agricultura climáticamente inteligente, entre otros), que proveen de servicios ecosistémicos vitales para el desarrollo de la vida del ser humano, no solo por los bienes que puede ofertar para suplir las necesidades de la población, sino también por la capacidad de regular y soportar funciones para el desarrollo de la vida en el planeta (Lewis et al., 2019; Owethu and Buschke, 2019). Se resalta el papel del árbol y la biodiversidad vegetal en el control de deslizamientos de tierra, fortaleciendo los servicios regulatorios que disminuyen las posibilidades de desastres en sistemas agroalimentarios Kobayashi and Mori, 2017), dando paso a la resiliencia y sustentabilidad de la producción agropecuaria comunitaria.

Aunque los SSE evidencian grandes ventajas para el desarrollo regional y de una nación, también es de anotarse algunas desventajas, tales como la falta de articulación con el sector del gobierno, donde las entidades no realizan la aplicación de las políticas establecidas, de tal manera que no hay retroalimentación entre los componentes del sistema (Ostrom, 1990). 


\section{CONCLUSIONES}

Los sistemas socio ecológicos han evolucionado desde el año 1971, bajo un motor determinado por la necesidad de integrar aspectos sociales, económicos y ambientales, con el fin de mitigar los impactos antrópicos mediante modelos que prevean el efecto de las actividades humanas sobre el medio y de esta manera transitar hacia procesos de gobernanza del territorio y manejo de los recursos naturales; herramienta clave para la gestión de los servicios ecosistémicos.

Los sistemas socio ecológicos permiten generar modelos a diferentes escalas productivas (médicas, industriales, etc.) para consolidar redes, relaciones y actividades entre la comunidad, el estado y la oferta ambiental; el mayor desarrollo de los SSE se ha generado en la agricultura, la cual ha evolucionado paralelamente con la presencia del ser humano en la tierra, dada la dependencia entre necesidad de alimento para el hombre y este como gestor del suelo para la producción de comida.

Los SSE contribuyen a la consolidación de redes alimentarias locales, se desarrollan inicialmente en nichos locales dentro de un régimen alimentario determinado, induciendo cambios socio-ecológicos a nivel local y tienen el potencial de fomentar transformaciones más amplias del sistema, adoptando el concepto de soberanía alimentaria, que permita la participación democrática y el compromiso cívico en la producción de alimentos y establezca nuevas relaciones que eviten la desigualdad social (seres humanos y naturaleza).

\section{AGRADECIMIENTOS}

Los autores agradecen a sus respectivas instituciones por el apoyo otorgado para la elaboración del presente documento.

\section{REFERENCIAS}

ACOSTA, CRISTINA; ORTEGA, MARIANA; BUNSEN, TILL; KOIRALA, BINOD PRASAD; GHORBANI, AMINEH. Facilitating energy transition through energy commons: An application of socio-ecological systems framework for integrated community energy systems. Sustainability, v. 10, n. 2, 2018, p. 366. https://doi.org/10.3390/su10020366

ALCAYNA, TILLY; BOLLETTINO, VICENZO; DY, PHILIP; VINCK, PATRICK. Resilience and disaster trends in the Philippines: opportunities for national and local capacity building. PLoS currents, v. 8, 2016. https://doi.org/10.1371/currents.dis.4aObc960866e53bd6357ac135d740846

ARARAL Jr, EDUARDO. What explains collective action in the commons? Theory and evidence from the Philippines. World development, v. 37, n. 3, 2009, p. 687-697. https://doi.org/10.1016/j.worlddev.2008.08.002

AZAR, CHRISTIAN; HOLMBERG, JOHN; LINDGREN, KRISTIAN. Socio-ecological indicators for sustainability. Ecological economics, v. 18, n. 2, 1996, p. 89-112. https://doi.org/10.1016/0921-8009(96)00028-6

BABAI, DÁNIEL; TÓTH, ANTONIA; SZENTIRMAI, IISTVÁN; BIRÓ, MARIANNA; MÁTÉ, AANDRÁS; DEMETER, LÁSLÓ; SZÉPLIGETI, MÁTYÁS; VARGA, ANNA; MOLNÁR, ABEL; KUN, RÓBERT; MOLNÁR, ZSOLT. Do conservation and agri-environmental regulations effectively support traditional small-scale farming in East-Central European cultural landscapes? Biodiversity and conservation, v. 24, n. 13, 2015, p. 3305-3327. https://doi.org/10.1007/s10531-015-0971-z

BARDSLEY, DOUGLAS. Risk alleviation via in situ agrobiodiversity conservation: drawing from experiences in Switzerland, Turkey and Nepal. Agriculture, Ecosystems \& Environment, v. 99, n. 1-3, 2003, p. 149-157. https://doi.org/10.1016/S0167-8809(03)00151-8 
BARDSLEY, DOUGLAS K.; BARDSLEY, ANNETTE M. Organising for socio-ecological resilience: The roles of the mountain farmer cooperative Genossenschaft Gran Alpin in Graubünden, Switzerland. Ecological Economics, v. 98, 2014, p. 11-21. https://doi.org/10.1016/j.ecolecon.2013.12.004

BARTON, JOHN; HASLETT, TIM. Analysis, synthesis, systems thinking and the scientific method: rediscovering the importance of open systems. Systems Research and Behavioral Science: The Official Journal of the International Federation for Systems Research, v. 24, n. 2, 2007, p. 143-155. https://doi.org/10.1002/sres.816

BAUDOUIN, YVES; RAYMOND, MICHEL; I DIALLO, ALPHA-PALLÉ. Modélisation de la dynamique des systèmes socio-écologiques en vue de l'élaboration d'un cadre de référence pour la durabilité environnementale et territoriale: application au massif du Fouta-Djalon (Guinée). European Journal of Geography, v. 1, 2014. https://doi.org/10.4000/cybergeo.26296

BEDDOE, RACHAEL; COSTANZA, ROBERT;FARLEY, JOSHUA; GARZA, ERIC; KENT,JENNIFER; KUBISZEWSKI, IDA; MARTÍNEZ, LUZ; MCCOWEN, TRACY; MURPHY, KATHLEEN; MYERS, NORMAN; OGDEN, ZACH; STAPLETON, KEVIN; WOODWARD, JOHN. Overcoming systemic roadblocks to sustainability: The evolutionary redesign of worldviews, institutions, and technologies. Proceedings of the National Academy of Sciences, v. 106, n. 8, 2009, p. 2483-2489. https://doi.org/10.1073/pnas.0812570106

BURKART, STEFAN; DÍAZ, MANUEL-FRANCISCO; ENCISO-VALENCIA, KAREN; URREA-BENITEZ, JOSÉLUIS; CHARRY-CAMACHO, ANDRÉS; TRIANA ÃNGEL, NATALIA. COVID-19 and the bovine livestock sector in Colombia: Current and potential developments, impacts and mitigation options. Cali (Colombia): International Center for Tropical Agriculture (CIAT), 2020, 36 p.

CARLSON, ULRIKA; HOLMBERG, JOHN; BERNDES, GORAN. Socio-ecological indicators for sustainability for Gotland, Sweden. Doktorsavhandlingar vid Chalmers Tekniska Hogskola, v. 1635, 2000, p. 1-62.

CARSON, RACHEL. Silent Spring. Hamilton. London (United Kingdom): 1963, p. 409.

CHERKASHIN, A.K.; LESHCHENKO, YA, A. Mathematical modeling and quantitative analysis of the demographic and ecological aspects of Russian supermortality. Mathematical Modelling of Natural Phenomena, v. 5, n. 6, 2010, p. 243-258. https://doi.org/10.1051/mmnp/20105611

CIFTCIOGLU, GULAY-CETINKAYA. Assessment of the resilience of socio-ecological production landscapes and seascapes: A case study from Lefke Region of North Cyprus. Ecological indicators, v. 73, 2017, p. 128-138. https://doi.org/10.1016/j.ecolind.2016.09.036

CHILE. COMISIÓN ECONÓMICA PARA AMÉRICA LATINA Y EL CARIBE (CEPAL). América Latina y el Caribe ante la pandemia del COVID-19: efectos económicos y sociales, Informe Especial COVID-19 No. 1. Economic Commission for Latin America and the Caribbean (CEPAL). Santiago (Chile): 2020, 19 p.

COMMONER, BARRY. Energía, medio ambiente y economía. Transición. Economía, trabajo, sociedad, v. 3, n. 1 , 1978, p. 13-16.

COMMONER, BARRY. La escasez de energía. Barcelona (España): Plaza y Janés, 1977.

COSTON-GUARINI, J.; GUARINI, J.M.; HINZ, SHAWN; WILSON, JEFF; CHAUVAUD, LAURENT. A roadmap for a quantitative ecosystem-based environmental impact assessment. ICES Journal of Marine Science, v. 74 n. 7, 2017, p. 2012-2023. https://doi.org/10.1093/icesjms/fsx015

COTE, MURIEL; NIGHTINGALE, ANDREA J. Resilience thinking meets social theory: situating social change in socio-ecological systems (SES) research. Progress in human geography, v. 36, n. 4, 2012, p. 475-489. https://doi.org/10.1177/0309132511425708

CRAIG, ROBIN K. Stationarity is dead e long live transformation: five principles for climate change adaptation law. The Harvard environmental law review, v. 34, n. 1, 2010, p. 9-28.

CRESPO-PÉREZ, VERÓNICA; REBAUDO, FRANÇOIS; SILVAIN, JEAN-FRANÇOIS; DANGLES, OLIVIER. Modeling invasive species spread in complex landscapes: the case of potato moth in Ecuador. Landscape ecology, v. 26, n. 10, 2011, p. 1447-1461. https://doi.org/10.1007/s10980-011-9649-4 
CROOK, J.H.; ELLIS, J.E.; GOSS-CUSTARD, J.D. Mammalian social systems: structure and function. Animal Behaviour, v. 24, n. 2, 1976, p. 261-274. https://doi.org/10.1016/S0003-3472(76)80035-8

CRUTZEN, PAUL J.; STOERMER, EUGENE F. The Anthropocene. Global Change Newsletter, v. 41, 2000, p. 17-18. CRUZ-PALACIOS, M.; SANTOS, EDD; VELÁSQUEZ-CERVANTES, M.A.; LEÓN-JUÁREZ, M. COVID-19, una emergencia de salud pública mundial. Revista Clínica Española, 2020. https://doi.org/10.1016/j.rce.2020.03.001

CUMMING, GRAEME S. Conceptual background on social-ecological systems and resilience. Spatial Resilience in Social-Ecological Systems. Dordrecht (Germany): Springer, 2011, p. 7-33. https://doi.org/10.1007/978-94-007-0307-0_2

CUMMINS, VALERIE; MCKENNA, JOHN. The potential role of sustainability science in coastal zone management. Ocean \& Coastal Management, v. 53, n. 12, 2010, p. 796-804. https://doi.org/10.1016/j.ocecoaman.2010.10.019

DANG, KINH-BAC; WINDHORST, WILHELM; BURKHARD, BENJAMIN; MÜLLER, FELIX. A Bayesian Belief Network-Based approach to link ecosystem functions with rice provisioning ecosystem services. Ecological Indicators, v. 100, 2019, p. 30-44. https://doi.org/10.1016/j.ecolind.2018.04.055

DAVIES, THOMAS; EVERARD, MARK; HORSWELL, MICHAEL. Community-based groundwater and ecosystem restoration in semi-arid north Rajasthan (3): Evidence from remote sensing. Ecosystem Services, v. 21, 2016. https://doi.org/20-30. 10.1016/j.ecoser.2016.07.007 2212-0416

DONADIEU, PIERRE. Reference systems in restoration ecology. Revue d'Ecologie (France). AGRIS, v.1, n. 9, 2002, p. 109-119.

DWYER, PETER D.; MINNEGAL, MONICA. Hunting in lowland, tropical rain forest: Towards a model of non-agricultural subsistence. Human Ecology, v. 19, n. 2, 1991, p. 187-212.

ELLEN, R.F. Problems and progress in the ethnographic analysis of small scale human ecosystems. Man, 1978, p. 290-303.

FERNÁNDEZ-REYES, ROGELIO. El Acuerdo de París y el cambio transformacional. Papeles de relaciones ecosociales y cambio global, v. 132, 2016, p. 101-114.

FRUEH-MUELLER, ANDREA; KRIPPES, CHRISTIAN; HOTES, STEFAN; BREUER, LUTZ; KOELLNER, THOMAS; WOLTERS, VOLKMAR. Spatial correlation of agri-environmental measures with high levels of ecosystem services. Ecological indicators, v. 84, 2018, p. 364-370. https://doi.org/10.1016/j.ecolind.2017.09.008

FULTON, ELIZABETH A.; JONES, T.; BOSCHETTI, FABIO; SPORCIC, M.; DE LA MARE, WILLIAM; SYME, GEOFFREY J; DZIDIC, PETA; GORTON, R.; LITTLE, L.R.; DAMBACHER, G.; CHAPMAN, KELLY. A multimodel approach to stakeholder engagement in complex environmental problems. Ecu Publications 2011, v. 1, p. 3098-3104.

GALLOPÍN, GILBERTO. El desarrollo sostenible desde una perspectiva sistémica. Sostenible, 2010, v. 11, 17-35.

GALLOPÍN, GILBERTO C. Human dimensions of global change: linking the global and the local processes. International Social Science Journal, v. 130, 1991.

GALLOPÍN, GILBERTO C. Linkages between vulnerability, resilience, and adaptive capacity. Global environmental change, v. 16, n. 3, 2006, p. 293-303.

https://doi.org/10.1016/j.gloenvcha.2006.02.004

GARMENDIA, ENEKO; STAGL, SIGRID. Public participation for sustainability and social learning: Concepts and lessons from three case studies in Europe. Ecological economics, v. 69, n. 8, 2010, p. 1712-1722. https://doi.org/10.1016/j.ecolecon.2010.03.027

GIRARDIN, OLIVIER; DAO, DAOUDA; KOUDOU, BENJAMIN G.; ESSÉ, CLĖMENCE; CISSÉ, GUÉLADIO; YAO, TANO; N'GORAN, ELIÈZER K.; TSCHANNEN, ANDREAS B.; BORDMANN, GÈRARD; LEHMANN, BERNARD; NSABIMANA, CHRISTIAN; KEISER, JENNIFER; KILLEN, GERRY F.; SINGER, BURTON H.; TANNER, MARCEL; UTZINGER, JHURG. Opportunities and limiting factors of intensive vegetable farming in malaria endemic Côte d'Ivoire. Acta Tropica, v. 89, n. 2, 2004, p. 109-123.

https://doi.org/10.1016/j.actatropica.2003.08.004 
GOODMAN, DAVID; DuPUIS E., MELANIE. Knowing food and growing food: beyond the productionconsumption debate in the sociology of agriculture. Sociologia ruralis, v. 42, n. 1, 2002, p. 5-22. https://doi.org/10.1111/1467-9523.00199

GROVE, THURMAN L.; EDWARDS, CLIVE A. Do we need a new developmental paradigm? Agriculture, Ecosystems \& Environment, v. 46, n. 1-4, 1993, p. 135-145. https://doi.org/10.1016/0167-8809(93)90019-L

HARDIN, GARRET. The tragedy of the commons. Science, v.162, n. 3859, 1968, p. 1243-1248. https://doi.org/10.1126/science.162.3859.1243

HE, HONG S.; LEWIS, BERNARD J.; BAER, ADAM D.; NIGH, TIMOTHY A. Exploring linkages between people and rural landscapes at broad ecological scales. Landscape and urban planning, v. 97, n. 1, 2010, p. 49-57. https://doi.org/10.1016/j.landurbplan.2010.04.011

HODGE, IAN. The governance of rural land in a liberalised world. Journal of Agricultural Economics, v. 58, n. 3 , 2007, p. 409-432. https://doi.org/10.1111/j.1477-9552.2007.00124.x

HOLLING, CRAWFORD-STANLEY; GUNDERSON, LANCE H. Resilience and adaptive cycles. Panarchy: Understanding Transformations in Human and Natural Systems. Washington D.C. (United States of America): Island Press, 2002.

HOLMBERG, JOHN; ROBÈRT, KARL-HENRIK. Backcasting-A framework for strategic planning. International Journal of Sustainable Development \& World Ecology, v. 7, n. 4, 2000, p. 291-308. https://doi.org/10.1080/13504500009470049

HONG-PHUONG, LE-THI; BIESBROEK, G. ROBERT; WALS, ARJEN E. The interplay between social learning and adaptive capacity in climate change adaptation: A systematic review. NJAS-Wageningen Journal of Life Sciences, v. 82, 2017, p. 1-9. https://doi.org/10.1016/j.njas.2017.05.001

HORNBORG, ALF. Zero-sum world: challenges in conceptualizing environmental load displacement and ecologically unequal exchange in the world-system. International Journal of Comparative Sociology, v. 50, n. 3-4, 2009, p. 237-262. https://doi.org/10.1177/0020715209105141

JAMES, CLIVE. Global Status of Commercialized Transgenic Crops. ISAAA Briefs No. 21. Ithaca, New York (United States of America): 2000.

JAMES, P.; ASHLEY, J.; EVANS, A. Ecological networks: Connecting environmental, economic and social systems? Landscape Research, v. 25, n. 3, 2000, p. 345-353. https://doi.org/10.1080/713684679

JIAO, YUANMEI; LIANG, LUOHUI. Strengthening the socio-ecological resilience of forest-dependent communities: The case of the Hani Rice Terraces in Yunnan, China. Forest Policy and Economics, v. 22, 2012, p. 53-59.

https://doi.org/10.1016/j.forpol.2012.04.004

JOUN, YOUNG P. Information requirement for socio-ecological models. The Annals of Regional Science, v. 5, n. 1, 1971, p. 25-32.

KANSIIME, MONICA K.; MASTENBROEK, ASTRID. Enhancing resilience of farmer seed system to climateinduced stresses: Insights from a case study in West Nile region, Uganda. Journal of Rural Studies, v. 47, 2016, p. 220-230. https://doi.org/10.1016/j.jrurstud.2016.08.004

KAUFMAN, DANIEL. Hunters-Gatherers of the Levantine Epipalaeolithic: The Socioecological Origins of Sedentism. Journal of Mediterranean Archaeology, v. 5, n. 2, 1992, p. 165-201. https://doi.org/10.1558/jmea.v5i1.165

KNOX, ANDREW; MYKHAYLOVA, NATALIA; EVANS, GREG J.; LEE, COLIN; KARNEY, BRYAN; BROOK, JEFFREY. The expanding scope of air pollution monitoring can facilitate sustainable development. Science of the total environment, v. 448, 2013, p. 189-196.

https://doi.org/10.1016/j.scitotenv.2012.07.096 
KOBAYASHI, YUTA; MORI, AKIRA S. The potential role of tree diversity in reducing shallow landslide risk. Environmental management, v. 59, n. 5, 2017, p. 807-815. https://doi.org/10.1007/s00267-017-0820-9

LAMBIN, ERIC F.; MEYFROIDT, PATRICK. Land use transitions: Socio-ecological feedback versus socioeconomic change. Land use policy, v. 27, n. 2, 2010, p. 108-118. https://doi.org/10.1016/j.landusepol.2009.09.003

LEVIN, SIMON A. Fragile dominion complexity and the commons. American Journal of Agricultural Economics, v. 83, n. 2, 2001, p. 246-247. https://doi.org/10.1111/1467-8276.t01-1-00151

LEWIS, CASEY L.; GRANEK, ELISE F.; NIELSEN-PINCUS, MAX. Assessing local attitudes and perceptions of non-native species to inform management of novel ecosystems. Biological Invasions, v. 21, n. 3, 2019, p. 961-982. https://doi.org/10.1007/s10530-018-1875-0

LUTZ, JULIANA. Do local food networks foster socio-ecological transitions towards food sovereignty? Learning from real place experiences. Sustainability, v. 5, n. 11, 2013, p. 4778-4796. https://doi.org/10.3390/su5114778

MACLEOD, CHRISTOPHER. What can we learn from systems based Approaches: from systems biology to earth systems science? 5th International Congress On Environmental Modelling And Software (Ottawa, Ontario, Canada, 2010). Memories, Ottawa (Canada): 2010, p. 1-9.

MAHMOODI, HASSAN; SHAGHAGHI, ABDOLREZA. Barriers and gateways to adapt standards of the Health Promoting Hospitals: a rigorous consolidation of the global research evidence. International Journal of Health Promotion and Education, v. 57, n. 5, 2019, p. 235-255. https://doi.org/10.1080/14635240.2019.1610025

MCKENZIE, SEAN; PARKINSON, HILARY; MANGOLD, JANE; BURROWS, MARY; AHMED, SELENA; MENALLED, FABIAN. Perceptions, Experiences, and Priorities Supporting Agroecosystem Management Decisions Differ among Agricultural Producers, Consultants, and Researchers. Sustainability, v. 10, n. 11, 2018, p. 4096. https://doi.org/10.3390/su10114096

McNALLY, AMY; MAGEE, DARRIN; WOLF, AARON-T. Hydropower and sustainability: Resilience and vulnerability in China's powersheds. Journal of environmental management, v. 90, 2009, p. S286-S293. https://doi.org/10.1016/j.jenvman.2008.07.029

MARTÍNEZ-FERNÁNDEZ, JULIA; ESTEVE-SELMA, MIGUEL-ÁNGEL;BAÑOS-GONZÁLEZ, ISABEL;CARREÑO, FRANCISCA; MORENO, ÀNGELES. Sustainability of Mediterranean irrigated agro-landscapes. Ecological Modelling, v. 248, 2013, p. 11-19. https://doi.org/10.1016/j.ecolmodel.2012.09.018

MATTHEWS, ROBIN; POLHILL, GARY; GILBERT, NIGEL; ROACH, ALAN. Integrating agent-based social models and biophysical models. MODSIM05-International Congress on Modelling and Simulation: Advances and Applications for Management and Decision Making, Proceedings (Canberra, Australia,2005). Memories, Canberra (Australia): 2005, p. p. 1617-1623.

MILESTAD, REBECKA; DARNHOFER, IKA. Building farm resilience: The prospects and challenges of organic farming. Journal of sustainable agriculture, v. 22, n. 3, 2003, p. 81-97. https://doi.org/10.1300/J064v22n03_09

NAUGHTON-TREVES, L. Whose animals? A history of property rights to wildlife in Toro, western Uganda. Land Degradation \& Development, v. 10, n. 4, 1999, p. 311-328. https://doi.org/10.1002/(SICI)1099-145X(199907/08)10:4<311::AID-LDR362>3.0.CO;2-3

NEGI, VIKRAM S.; MAIKHURI, RAO K. Socio-ecological and religious perspective of agrobiodiversity conservation: issues, concern and priority for sustainable agriculture, Central Himalaya. Journal of agricultural and environmental ethics, v. 26, n. 2, 2013, p. 491-512.

https://doi.org/10.1007/s10806-012-9386-y

NILSSON, JIM; BERGSTRÖM, SÖREN. Indicators for the assessment of ecological and economic consequences of municipal policies for resource use. Ecological Economics, v. 14, n. 3, 1995, p. 175-184.

https://doi.org/10.1016/0921-8009(95)00013-Y 
NUNES, ANDRÉ-VALLE; GUARIENTO, RAFAEL-DETTOGNI; SANTOS, BRÁULIO- ALMEIDA; FISCHER, ERICH. Wild meat sharing among non-indigenous people in the southwestern Amazon. Behavioral ecology and sociobiology, v. 73, n. 2, 2019, p. 26. https://doi.org/10.1007/s00265-018-2628-x

O'NEAL-CAMPBELL, MICHAEL. The impact of small-scale wood harvesting on neem Azadirachta indica A. Juss stands in the coastal savanna of Ghana. Area, v. 37, n. 1, 2005, p. 8-16. https://doi.org/10.1111/j.1475-4762.2005.00611.x

UNITES STATES OF AMERICA. ORGANIZACIÓN DE LAS NACIONES UNIDAS (ONU). De la Convención del Protocolo de Kyoto. Marco de Las Naciones Unidas sobre el cambio climático. Kyoto (Japón): Naciones Unidas, v. 25, 1998, p. 27.

UNITES STATES OF AMERICA. ORGANIZACIÓN DE LAS NACIONES UNIDAS (ONU). Declaración del Milenio. Asamblea General del Quincuagésimo periodo de sesiones. Nueva York (United States of Ameerica): v.13, 2000

UNITES STATES OF AMERICA. ORGANIZACIÓN DE LAS NACIONES UNIDAS (ONU). Programa para el medio ambiente. Coronavirus: ¿llegó para quedarse?. 2020. https://www.unenvironment.org/es/noticias-yreportajes/reportajes/coronavirus-llego-para-quedarse [consultado junio 8 de 2020].

ORO, KOUHEI. The development of beef cattle breeding in Chiburi-jima Island, Shimane Prefecture. Japanese Journal of Human Geography, v. 52, n. 6, 2000, p. 575-595. https://doi.org/10.4200/jjhg1948.52.575

OSTROM, ELINOR. A general framework for analyzing Sustainability of Social-ecological systems. Science, v. 325, 2009, p.419- 422. https://doi.org/10.1126/science.1172133

OSTROM, ELINOR. El Gobierno de los bienes comunes. La evolución de las instituciones de acción colectiva. México D.F. (México): Universidad Nacional Autónoma de México (UNAM), 1990.

OSTROM, ELINOR. El gobierno de los bienes comunes - La evolución de las Instituciones de acción colectiva. 2 ed. México D.F. (México): UNAM-CRIM-FCE, 2011.

OWETHU PANTSHWA, A.; BUSCHKE, FALKO T. Ecosystem services and ecological degradation of communal wetlands in a South African biodiversity hotspot. Royal Society open science, v. 6, n. 6, 2019., p. 181770. https://doi.org/10.1098/rsos.181770

PADMANABHAN, MARTINA. Women and men as conservers, users and managers of agrobiodiversity: A feminist social-ecological approach. The Journal of Socio-Economics, v. 40, n. 6, 2011, p. 968-976. https://doi.org/10.1016/j.socec.2011.08.021

PLUT, DUSAN. Teoretično-metodološki vidiki sistemskega geografskega raziskovanja pokrajinske degradacije. Dela, v. 11, 1995, p. 181-205.

RAMAKRISHNAN, PALAYANOOR-SIVASWAMY. Linking natural resource management with sustainable development of traditional mountain societies. Tropical Ecology, v. 44, n. 1, 2003, 43-54.

REDMAN, CHARLES L.; KINZIG, ANN P. Resilience of past landscapes: resilience theory, society, and the longue durée. Conservation ecology, v. 7, n. 1, 2003.

RUDDIMAN, WILLIAM F.; KUTZBACH, JOHN E. Plateau uplift and climate change. Scientific American, v. 264, 1991, p. 66-74.

https://doi.org/10.1017/S0263593300020812

RUIZ-BALLESTEROS, ESTEBAN; GUAL, MIGUEL A. The emergence of new commons. Human Ecology, v. 40, n. 6, 2012, p. 847-862.

https://doi.org/10.1007/s10745-012-9540-1

SANCHO, ROSA. Indicadores bibliométricos utilizados en la evaluación de la ciencia y la tecnología. Revisión bibliográfica. Revista española de documentación científica, v. 13, n. 3-4, 1990.

SCHUMACHER, ERNST-FRIEDRICH. Lo pequeño es hermoso. Madrid (España): Tursen/Hermann Blume,1978.

SESSA, CARLO; RICCI, ANDREA. Working with and for the citizens. Innovation-The European Journal of Social Science Research, v. 23, n. 1, 2010, p. 49-60. https://doi.org/10.1080/13511611003791174 
SHOOSHTARIAN, SALMAN; RAJAGOPALAN, PRIYADARSINI. Study of thermal satisfaction in an Australian educational precinct. Building and Environment, v. 123, 2017, p. 119-132.

https://doi.org/10.1016/j.buildenv.2017.07.002

SWORD, WENDY. Accounting for presence of self: Reflections on doing qualitative research. Qualitative Health Research, v. 9, n. 2, 1999, p. 270-278. https://doi.org/10.1177/104973299129121839

SYME, GEOFFREY J.; DAMBACHER, J.M.; DZIDIC, PETA L.; FULTON, ELIZABETH A.; BOSCHETTI, FABIO. Integrating research, modelling and decision making: A network and knowledge processing approach for sustainable management of a coral reef. 18th World IMACS/MODSIM Congress (Cairns, Australia, 2009). Memories, Cairns (Australia): 2009, p. 2915-2921.

TEWARI, RITIKA. NAMAs, international support, and appropriateness: Reflections from COP 18. Mitigation Talks, v. 3, n. 4, 2013, p. 2-4.

THOMSON, ALAN J. Paradigm green-ai approaches to evaluating the economic consequences of changing environmental viewpoints. Ai Applications, v. 7, n. 4, 1993, p. 61-68.

TORRES-LEZAMA, ARMANDO; VILANOVA, EMILIO; RAMÍREZ-ANGULO, HIRMA. Guiding principles for small-scale forestry in a watershed of the Venezuelan Andes: constraints and opportunities. Small-scale Forestry, v. 8, n. 1, 2009, p. 77-93. https://doi.org/10.1007/s11842-008-9068-9

TRAVERS, HENRY; CLEMENTS, TOM; KEANE, AIDAN; MILNER-GULLAND, E.J. Incentives for cooperation: The effects of institutional controls on common pool resource extraction in Cambodia. Ecological Economics, v. 71, 2011, p. 151-161. https://doi.org/10.1016/j.ecolecon.2011.08.020

VAHANVATI, MITTUL. A novel framework for owner driven reconstruction projects to enhance disaster resilience in the long term. Disaster Prevention and Management: An International Journal, v. 27, n. 4, 2018, p. 421-446. https://doi.org/10.1108/DPM-11-2017-0285

VERBESSELT, JAN; ZEILEIS, ACHIM; HEROLD, MARTIN. Near real-time disturbance detection using satellite image time series. Remote Sensing of Environment, v. 123, 2012, p. 98-108. https://doi.org/10.1016/j.rse.2012.02.022

VERHOOG, REINIER; GHORBANI, AMINEH; DIJKEMA, GERARD. Modelling socio-ecological systems with MAIA: A biogas infrastructure simulation. Environmental Modelling \& Software, v. 81, 2016, p. 72-85. https://doi.org/10.1016/j.envsoft.2016.03.011

VITYAKON, PATMA; SUBHADHIRA, SUKAESINEE; LIMPINUNTANA, VIRIYA; SRILA, SOMJAI; TRELO-GES, VIDHAYA; SRIBOONLUE, VICHAI. From Forest to Farmfields. Japanese Journal of Southeast Asian Studies, v. 41, n. 4, 2004, p. 444-472. https://doi.org/10.20495/tak.41.4_444

WAYLEN, KERRY A.; BLACKSTOCK, KIRSTY L.; VAN HULST, FREDDY J.; DAMIAN, CARMEN; HORVÁTH, FERENC; JOHNSON, RICHARD K.; KANKA, ROBERT; KÜLVIK, MART; MACLEOD, CHRISTOPHER J.A.; MEISSNER, KRISTIAN; OPRINA-PAVELESCU, MIHAELA M.; PINO, JOAN; PRIMMER, EEVA; RÎSNNOVEANU, GETA; ŠATALOVÁ, BARBORA; SILANDER, JARI; ŠPULEROVÁ, JANA; SUŠKEVIČS, MONIKA; UYTVANCK, JANVAN. Policy-driven monitoring and evaluation: Does it support adaptive management of socio-ecological systems? Science of the Total Environment, v. 662, 2019, p. 373-384. https://doi.org/10.1016/j.scitotenv.2018.12.462

WESTERN, DAVID. The challenge of integrated rangeland monitoring: synthesis address. African Journal of Range and Forage Science, v. 21, n.2, 2004, p. 129-136. https://doi.org/10.2989/10220110409485844

WHITE, NORMAN-FREDERICK. Medical and graduate education in behavioral medicine and the evolution of health care. Annals of Behavioral Medicine, v. 10, n. 1, 1988, p. 23-29. https://doi.org/10.1207/s15324796abm1001_7 
WILLEBRAND, TOMAS; SANDSTRÖM, CAMILLA; LUNDGREN, TOMMY. Reaching for new perspectives on socioecological systems: exploring the possibilities for adaptive co-management in the Swedish mountain region. The International Journal of Biodiversity Science and Management, v. 2, n. 4, 2006, p. 359-369. https://doi.org/10.1080/17451590609618157

WORLD HEALTH ORGANIZATION (WHO). Regional Overview of Food Security in Latin America and the Caribbean: Towards healthier food environments that address all forms of malnutrition. Food \& Agriculture Organization, v.12, 2020, 135 p.

YOUNG, ORAN R.; BERKHOUT, FRANS; GALLOPIN, GILBERTO C.; JANSSEN, MARCO A.; OSTROM, ELINOR; VAN DER LEEUW, SANDER. The globalization of socio-ecological systems: an agenda for scientific research. Global Environmental Change, v. 16, n. 3, 2006, p. 304-316. https://doi.org/10.1016/j.gloenvcha.2006.03.004

ZACHOS, LOUIS-G. Research output evaluation of two university departments in Greece with the use of bibliometric indicators. Scientometrics, v. 21, n. 2, 1991, p. 195-221. https://doi.org/10.1007/BF02017569 